\title{
Rastreamento Pré-natal de Anormalidades Cardíacas: Papel da Ultra-sonogrrafia Obstétrica de Rotina
}

\author{
Prenatal Screening of Cardiac Abnormalities: The Role of Routine \\ Obstetrical Ultrasound
}

Tzvi Bacaltchuk, Paula Antunes, Paulo Zielinsky

\section{RESLMO}

Objetivo: avaliar o papel da ultra-sonografia obstétrica de rotina no rastreamento pré-natal de cardiopatias congênitas ou arritmias graves e os fatores envolvidos na sua acurácia.

Métodos: a amostra foi constituida de 77 neonatos ou lactentes internados no Instituto de Cardiologia do Rio Grande do Sul no periodo de maio a outubro de 2000, com diagnóstico pós-natal confirmado de cardiopatia estrutural ou arritmia grave, que tinham sido submetidos, durante a vida fetal, a pelo menos uma ultra-sonografia obstétrica após a $18^{a}$ semana de gestação. Após consentimento informado, um questionário padronizado foi aplicado. As variáveis categóricas (tipo de cardiopatia e a acessibilidade ecográfica para o diagnóstico, alteração do ritmo cardíaco, número de gestações, paridade, abortamentos prévios, estado civil das gestantes, tipo de parto, área de internação hospitalar, sexo, tipo de serviço onde foi realizado o pré-natal, indicação da ultra-sonografia obstétrica, número de ultra-sonografias realizadas, tipo de serviço onde foi realizada a ultra-sonografia obstétrica, local onde foi realizada a ultra-sonografia obstétrica, renda familiar, escolaridade materna e escolaridade paterna) foram comparadas pelo teste do $\chi^{2}$ ou pelo teste exato de Fisher e um modelo de regressão logística foi utilizado para determinar variáveis independentes eventualmente envolvidas na suspeita pré-natal de cardiopatia.

Resultados: em 19 pacientes (24,7\%), a ultra-sonografia obstétrica foi capaz de levantar suspeita de anormalidades estruturais ou de arritmias. Ao serem consideradas apenas as cardiopatias congênitas, esta taxa foi de 19,2\% (14/73). Em 73,7\% dos pacientes com suspeita de anormalidades cardiacas durante a ultra-sonografia obstétrica, as cardiopatias suspeitadas eram acessiveis ao corte de 4 câmaras isolado. Observou-se que 26,3\% das crianças com suspeita pré-natal de cardiopatia apresentaram arritmias durante o estudo ecográfico, ao passo que apenas 3,4\% dos pacientes sem suspeita pré-natal apresentaram alterações do ritmo ( $p=0,009)$. Constituíram fatores comparativos significantes entre o grupo com suspeita pré-natal e o sem suspeita a paridade $(p=0,029)$, o parto cesáreo $(p=0,006)$, a internação em unidade de tratamento intensivo $(p=0,046)$ e a escolaridade paterna $(p=0,014)$. À análise multivariada, apenas a presença de alteração do ritmo cardíaco durante a ultra-sonografia obstétrica mostrou-se como variável independente associada à suspeita pré-natal de anormalidade cardiaca.

Conclusões: a ultra-sonografia obstétrica de rotina ainda tem sido subutilizada no rastreamento pré-natal de cardiopatias congênitas. O treinamento dirigido dos ultrasonografistas e a conscientização do meio obstétrico e da própria população podem ser os instrumentos para aumentar a eficácia deste método.

PALAVRAS-CHAVE: Ultra-sonografia pré-natal. Cardiopatias congênitas. Diagnóstico prénatal. Coração fetal.

Unidade de Cardiologia Fetal do Instituto de Cardiologia do Rio Grande do Sul - Fundação Universitária de Cardiologia. Universidade Federal do Rio Grande do Sul. - Programa de Pós-Graduação: Pediatria

Correspondência:

Tzvi Bacaltchuk - Unidade de Pesquisa do IC/FUC

Av. Princesa Isabel, 395 - Santana

90620-001 - Porto Alegre - RS

Fone/Fax: (51) 230-3600 R. 3777 - e-mail: pesquisa@cardnet.tche.br 
Introdução

Vários estudos com o objetivo de avaliar os possiveis benefícios e a sensibilidade da ultrasonografia obstétrica no acompanhamento pré-natal de rotina foram realizados nos últimos anos ${ }^{1}$, mas são muito escassos os trabalhos que avaliam seu papel na detecção ou suspeita de anormalidades cardíacas.

A menos que um rigoroso rastreamento seja feito durante a gestação, as cardiopatias congênitas não serão identificadas até o nascimento. Entre 20 e $32 \%$ das mortes perinatais são devidas a doenças congênitas do coração ${ }^{2-5}$, mas este número provavelmente é maior se levarmos em conta o período gestacional, principalmente se considerarmos o fato de que cardiopatias congênitas são responsáveis por número expressivo de mortes fetais ${ }^{2}$. Estima-se que 15 a 20\% dos abortos espontâneos sejam decorrentes de anomalias cardiacas ${ }^{6}$.

As cardiopatias congênitas são a terceira causa de morte no período neonatal e a terceira causa específica de mortalidade infantil no Rio Grande do Sul (coeficiente de mortalidade específica: 94,6/1000 ${ }^{7}$. Surpreendentemente, estas mesmas anomalias cardíacas estão entre as lesões que menos freqüentemente são diagnosticadas nas triagens durante o período pré-natal ${ }^{8}$. Segundo dados do Ministério da Saúde, há no Rio Grande do Sul cerca de 180.000 nascimentos por ano, levando a uma estimativa de 2000 casos novos de cardiopatia por ano neste estado ${ }^{9}$. As crianças com cardiopatias congênitas utilizam 25 a 30\% dos leitos nas unidades de tratamento intensivo pediátricas e neonatais, consumindo grande parte dos recursos existentes, destinados à assistência desta faixa etária, para todas as outras doenças ${ }^{10}$.

Não há controvérsias no que se refere à elevada prevalência de anormalidades cardíacas fetais em gestações com risco aumentado, seja por fatores maternos ou fetais (história familiar de cardiopatia, exposição a teratógenos, exposição a drogas que causem sofrimento fetal, distúrbios metabólicos maternos, idade maior que 35 anos, infecções virais, colagenoses, polidrâmnio, oligoâmnio, translucência nucal aumentada, anomalias extracardíacas, arritmias cardíacas, hidropisia fetal não-imune, crescimento intrauterino restrito, e suspeita de anomalia cardíaca ao exame ecográfico prévio) ${ }^{11}$. Entretanto, deve ser enfatizado o fato de que apenas $10 \%$ das cardiopatias congênitas ocorrem em grávidas com fatores de risco, o que implica afirmar que $90 \%$ das anormalidades cardiacas incidem na população geral, sem risco aumen$\operatorname{tado}^{6,9}$. Por isso, a única forma de detectar essas anomalias é o estudo sistemático do coração fetal, em todas as grávidas.
Este trabalho tem o objetivo de avaliar o papel da ultra-sonografia obstétrica de rotina na suspeita pré-natal de cardiopatias congênitas ou arritmias graves e os fatores envolvidos em sua acurácia.

\section{Métodos}

Este é um estudo transversal, em que o fator em estudo é a ultra-sonografia obstétrica de rotina e o desfecho a suspeita pré-natal de cardiopatia estrutural ou alteração grave do ritmo cardíaco fetal.

A amostra é constituída de 77 neonatos ou lactentes internados no Instituto de Cardiologia do Rio Grande do Sul, no período de maio a outubro de 2000, com diagnóstico pós-natal confirmado de cardiopatia congênita ou arritmia grave (taquiarritmia atrial ou bloqueio atrioventricular total), atendidos de forma seqüencial e não-intencional, que tenham sido submetidos, durante a vida fetal, a pelo menos uma ultra-sonografia obstétrica após a $18^{\mathrm{a}}$ semana de gestação.

Foram excluídas todas as crianças cujas mães não haviam sido submetidas a estudo ultrasonográfico obstétrico ou as que foram submetidas a este exame apenas antes da $18^{\mathrm{a}}$ semana de gestação. Também foram excluídas aquelas com diagnóstico de lesões isoladas de detecção pré-natal impossivel ou reconhecidamente difícil (persistência do canal arterial, comunicação interatrial tipo fossa oval, comunicação interventricular pequena e estenose pulmonar leve), assim como as que apresentavam outras alterações do ritmo cardíaco (extra-sístoles, taquicardia ou bradicardia sinusal).

Para a coleta dos dados, foi aplicado um questionário destinado a estabelecer as características demográficas, econômicas e sociais de cada caso, a presença de fatores de risco maternos ou fetais para cardiopatia congênita, o número de ecografias obstétricas a que a gestante foi submetida, o local onde esses exames foram realizados e a eventual suspeita pré-natal de cardiopatia ou arritmia grave a partir dos mesmos.

Uma vez internado um paciente durante o período de estudo (de maio a outubro de 2000), o autor era imediatamente comunicado, para que fossem avaliados os critérios de inclusão do mesmo no trabalho. Se incluído, a mãe (preferencialmente), o pai ou o responsável legal pela criança eram contatados pelo mesmo ou por um bolsista de Iniciação Científica participante do estudo. No caso de anuência, assinavam um termo de consentimento livre e esclarecido.

Todas as crianças tiveram seu diagnóstico estabelecido após anamnese, exame físico completo, eletrocardiograma, radiografia de coração e vasos da base e ecocardiograma uni/bidimensional 
com Doppler e mapeamento a cores. Quando necessário para complementação propedêutica ou intervenção terapêutica, foi também realizado cateterismo cardíaco, com estudo hemodinâmico e angiografia. Os casos submetidos a cirurgias corretivas ou aqueles falecidos e encaminhados a exame post mortem tiveram, ainda, confirmação anatômica direta do diagnóstico morfológico de sua cardiopatia.

O presente estudo foi aprovado pelo Comitê de Ética em Pesquisa do Instituto de Cardiologia do Rio Grande do Sul.

Os dados foram dispostos em um banco de dados do programa Epi-Info, versão 6.04 para análise descritiva, com cálculo de média, mediana e desvio padrão para cada variável. Na análise bivariada, as variáveis categóricas foram comparadas pelo teste do $\chi^{2}$ com correção de Yates ou pelo teste exato de Fisher, quando apropriado. Foi utilizado intervalo de confiança de $95 \%$ e alfa crítico de $5 \%$. Na comparação entre os dois grupos, aqueles parâmetros que se mostraram significantes ou com valores de $\mathrm{p}$ próximos a 0,05 , à análise bivariada, foram incluídos como variáveis explanatórias, em um modelo de regressão logística, utilizando o programa SPSS ("Statistical Package for Social Sciences”, versão 6.1.3), considerando como variável dependente a suspeita pré-natal de cardiopatia à ultra-sonografia obstétrica.

\section{Resultados}

A amostra foi constituída de 77 recém-nascidos e lactentes portadores de cardiopatias estruturais ou arritmias cujas mães informaram terem sido submetidas a ultra-sonografia obstétrica a partir da $18^{\mathrm{a}}$ semana de gestação.

A média de idade das mães participantes do trabalho foi de 27 anos e o número de ultrasonografias realizadas neste grupo de mães variou de 1 até 20 exames, com média de 4 exames por gestação.

Entre os 77 pacientes da amostra, 19 tiveram suspeição da anormalidade durante o estudo ultrasonográfico obstétrico, correspondendo a $24,7 \%$ da amostra. Como as quatro arritmias graves ( 3 casos de bloqueio atrioventricular total e 1 de taquicardia atrial sustentada) foram detectadas durante a ecografia de rotina, as cardiopatias estruturais foram suspeitadas em 14, correspondendo a 19,2\% dos casos. Em 14/19 pacientes com suspeita de anormalidade cardíaca à ultra-sonografia obstétrica $(73,7 \%)$, as cardiopatias podiam ser identificadas ao corte de 4 câmaras isolado (Tabela 1).

Tabela 1 - Diagnósticos dos pacientes estudados e frequência relativa dos casos com e sem suspeita pré-natal.

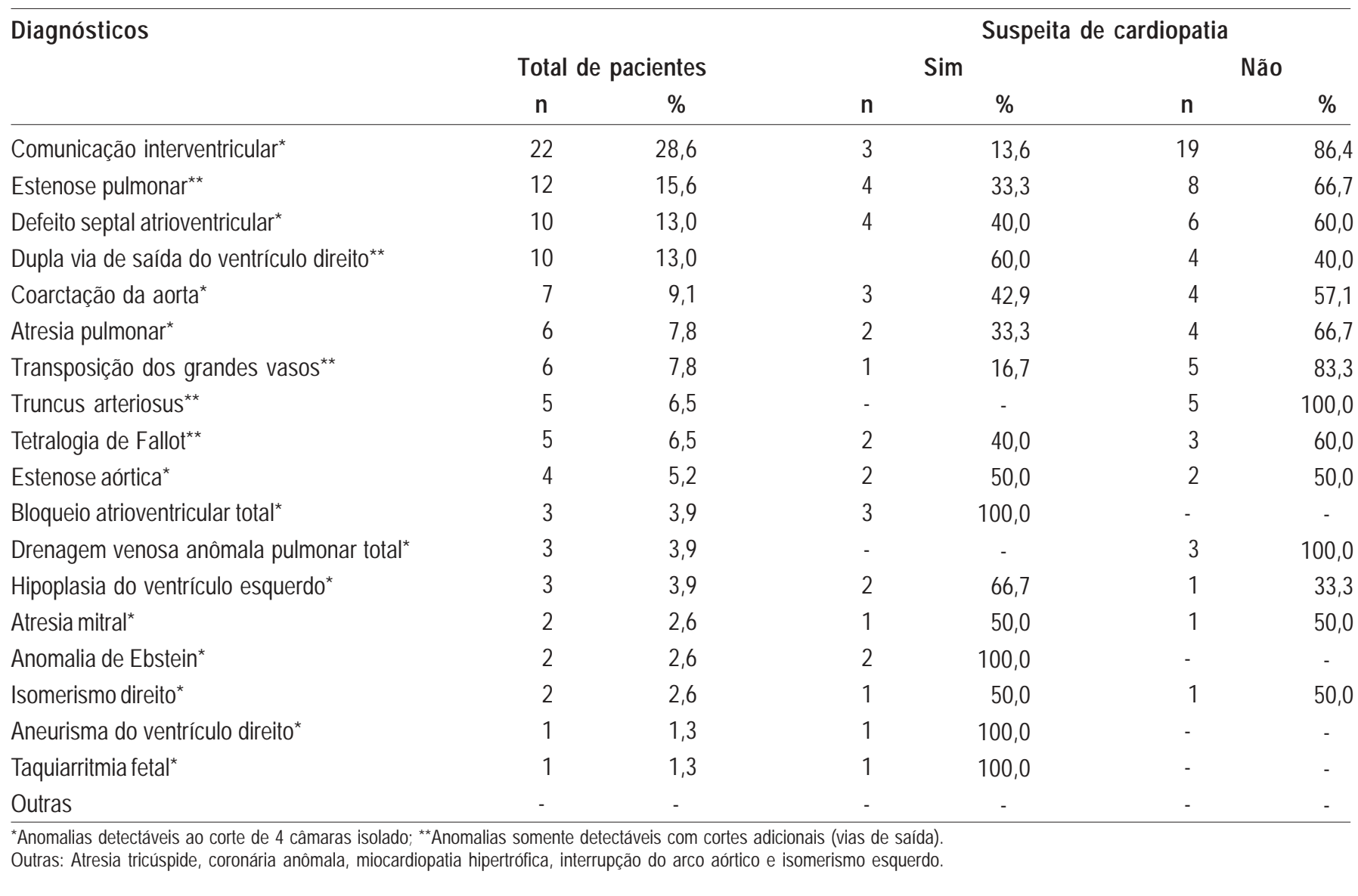


Observou-se que $26,3 \%$ das crianças com suspeita pré-natal de cardiopatia apresentaram arritmias durante o estudo ecográfico, ao passo que apenas $3,4 \%$ dos pacientes sem suspeita prénatal apresentaram alterações do ritmo $(\mathrm{p}=0,009)$.

No grupo com suspeita pré-natal de cardiopatia, $14 / 19(73,7 \%)$ eram primíparas, e no grupo sem suspeita, $24 / 58(41,4 \%)(p=0,029)$. No grupo com suspeita, apenas $18 / 19$ pacientes nasceram de parto cesáreo $(94,7 \%)$ e no grupo sem suspeita, 33/58 (56,9\%) ( $\mathrm{p}=0,006)$. Do grupo com suspeita, 16 de 19 pacientes $(84,2 \%)$ estavam internados na unidade de tratamento intensivo pediátrico, contra 32/58 das crianças sem suspeita $(55,2 \%)(p=0,046)$. No grupo com suspeita prénatal, $13 / 17$ dos pais $(76,5 \%)$ tinham $1^{\circ}$ grau completo ou mais, e no grupo sem suspeita, 22/57 $(38,6 \%)$ tinham este nivel de escolaridade $(\mathrm{p}=0,014)$. Não se mostraram significativos o número de gestações, a história de abortos prévios, o estado civil, o sexo dos pacientes, o tipo de serviço e a localidade em que foram realizados o pré-natal e a ultra-sonografia obstétrica, a indicação da ecografia, o número de ultra-sonografias realizadas, a renda familiar e a escolaridade materna.

O modelo de regressão logística, considerando como variáveis explanatórias o número de gestações, a paridade, as alterações do ritmo, o tipo de parto, a escolaridade materna e a escolaridade paterna, e como variável dependente a suspeita pré-natal de cardiopatia à ultra-sonografia obstétrica de rotina, mostrou que a única variável independente associada à suspeita de cardiopatia foi a alteração do ritmo cardíaco fetal durante a ecografia obstétrica.

\section{Discussão}

Deste grupo de 77 pacientes, apenas 19 tiveram despertada a suspeita de serem portadores de algum tipo de anormalidade cardíaca detectável à ultra-sonografia obstétrica de rotina, realizada após a $18^{\mathrm{a}}$ semana de gestação, o que corresponde a um quarto dos casos apenas. Excluindo-se as quatro arritmias graves, todas suspeitadas à ultrasonografia obstétrica, esta taxa cai para 19,2\%, consideradas apenas as anomalias estruturais.

Este resultado evidencia melhora no índice de detecção das anormalidades cardíacas no nosso meio, pois outro trabalho realizado na mesma instituição, em 1996, com características semelhantes, mostrou índice de suspeita de anormalidades cardíacas de $8 \%(5 / 80)$ das pacientes que se submeteram à ultra-sonografia obstétrica de rotina após a $18^{\mathrm{a}}$ semana de gestação ${ }^{12}$. Muitos fatores podem ter influenciado nesta melhora na acurácia da ultra-sonografia obstétrica de rotina, mas é possivel levantar a hipótese de que a curva de aprendizado seja o fator preponderante. Obviamente, as campanhas de conscientização da população no sentido de buscar mais freqüentemente o diagnóstico pré-natal de cardiopatias e a melhor compreensão da anatomia do coração fetal pelo ultra-sonografista têm seu papel. De qualquer maneira, índice de suspeita menor do que $20 \%$ de cardiopatias estruturais importantes a ponto de tornar necessária hospitalização é claramente insuficiente.

Dos 19 pacientes que tiveram despertada suspeita pré-natal de anormalidade cardíaca à ultrasonografia obstétrica, 14 (73,7\%) tinham doenças detectáveis com o uso do corte de 4 câmaras. As demais cardiopatias somente seriam detectáveis se fosse acrescentado um corte ultra-sonográfico que pudesse identificar as vias de saída dos ventrículos direito e esquerdo e as artérias pulmonares e aorta. Estes dados corroboram a hipótese levantada por outros autores ${ }^{1,2,13-22}$ de que a simples inclusão dos cortes de vias de saída ao exame ultra-sonográfico de rotina aumentaria a sensibilidade do método. Em estudo de 5967 fetos, foi descrita sensibilidade de $47 \%$ para o corte de 4 câmaras, que aumentou para $78 \%$ com a inclusão dos cortes de via de saída ventricular ${ }^{13}$.

Anormalidades do ritmo cardíaco fetal são fatores comuns de encaminhamento de gestantes para rastreamento de anormalidades estruturais do coração fetal. Dos pacientes avaliados neste estudo, 4 apresentaram alterações graves do ritmo cardíaco como anormalidade básica e isolada (3 casos de bloqueio atrioventricular total e 1 de taquicardia atrial sustentada), representando $5,2 \%$ dos casos e determinando, por este motivo, sua internação. Todos os quatro fetos tiveram sua arritmia detectada durante a ultra-sonografia obstétrica de rotina. Esta informação vem confirmar o observado por outros autores ${ }^{16,23}$, de que é possível detectar alterações do ritmo cardíaco pela ultra-sonografia obstétrica se for dedicada atenção especial por parte do examinador.

Esta idéia ficou reforçada ao se observar, neste trabalho, que mais de dois terços dos fetos com arritmia observada à ultra-sonografia obstétrica estavam no grupo com suspeita de cardiopatia, ao passo que no grupo sem arritmia, apenas $20 \%$ despertaram suspeita pré-natal de anormalidade cardíaca. Além disso, a análise multivariada demonstrou que a presença de alteração do ritmo cardíaco durante o exame ecográfico obstétrico de rotina é uma variável independente associada à suspeita pré-natal de anormalidade cardíaca. À luz destes achados, a ecocardiografia 
fetal completa deveria ser preconizada em todos os casos nos quais o obstetra detectasse algum tipo de alteração do ritmo cardíaco fetal.

Outro dado que se mostrou significante foi o que se refere ao número de filhos das famílias que participaram do estudo. Nota-se que quase três quartos das mães entrevistadas, em que foi despertada suspeita de anomalia cardíaca em seus fetos, eram primíparas $(73,7 \%)$. Especula-se que a atenção dedicada durante o estudo ultrasonográfico obstétrico nas primíparas pudesse ser maior, talvez estimulado pela maior preocupação da gestante.

Outro dado também pouco discutido na literatura refere-se ao tipo de parto oferecido às mães de crianças cardiopatas. Das crianças que tiveram sua cardiopatia detectada ainda intra-útero, apenas uma nasceu de parto normal, ao passo que $94,7 \%$ das crianças que tiveram suspeita de serem portadores de anomalia cardíaca nasceram de parto cesáreo. Este dado reflete a tendência de organizar melhor o tipo de atendimento dispensado durante o nascimento destas crianças, que assim tinha "hora marcada", com equipe multidisciplinar à disposição e vaga assegurada em unidade de tratamento intensivo neonatal ${ }^{6,11,24}$.

No que se refere à área de internação dos pacientes, observou-se que $84,2 \%$ dos pacientes do grupo que teve sua cardiopatia suspeitada estavam internados na UTI pediátrica e $89,7 \%$ dos pacientes que não tiveram nenhuma suspeita de anormalidade cardiaca congênita estavam internados em outra área do hospital, fora das unidades de tratamento intensivo, reforçando o fato de que foram os casos mais graves aqueles que chamaram a atenção do ultra-sonografista obstétrico, o que tem também sido comentado por outros autores $^{9,10}$.

O nível de escolaridade paterna mostrou associação significativa, no que se refere à suspeita de anormalidades cardíacas fetais durante a ultra-sonografia obstétrica de rotina. Note-se que $76,5 \%$ dos casos que despertaram suspeita de anormalidade cardíaca do feto tinham pais com nível de escolaridade maior que os pais do grupo que não despertou suspeita.

O fulcro da presente investigação, e que deve ser considerado como o mote fundamental para mudança de atitudes nos meios obstétrico e da ultra-sonografia, é a constatação de que, embora existam serviços qualificados que analisam o coração rotineiramente durante o exame ecográfico pré-natal, e assim estão instrumentalizados para a detecção de anormalidades, ainda é inaceitavelmente grande o número de cardiopatias que passam pela tela do ultra-sonografista sem levantar suspeita. Estes resultados têm reflexos dire- tos no nível de atenção pré-natal e na possibilidade de sucesso do tratamento cardiológico dos conceptos com cardiopatia e, portanto, podem representar o diferencial entre a vida e a morte no periodo perinatal. Por essas razões, a demonstração da subutilização da ultra-sonografia obstétrica no rastreamento pré-natal de anormalidades cardíacas enfatiza a necessidade de implementação de programas efetivos de treinamento de ecografistas para incluir a análise das estruturas cardíacas durante a ultra-sonografia obstétrica de rotina, além da conscientização do meio médico e da própria população.

\section{SUMMARY}

Purpose: to evaluate the role of routine obstetrical ultrasound scan in suspecting the presence of fetal congenital heart diseases and severe arrhythmias, as well as the factors involved in its accuracy.

Methods: the sample was made up of 77 neonates and infants hospitalized at the Institute of Cardiology of Rio Grande do Sul from May to October of 2000, with confirmed postnatal diagnosis of structural heart disease or severe arrhythmia, whose mothers had been submitted to at least one obstetrical ultrasound scan after 18 weeks of gestation. After informed consent, a customized standard questionnaire was used. Categorical variables were compared using $\chi^{2}$ test or Fisher's exact test and a logistic regression model was used to determine independent variables possibly involved in the prenatal suspicion of cardiac abnormalities.

Results: in 19 patients (24.6\%), obstetrical ultrasound was able to rise prenatal suspicion of structural or rhythm abnormalities. Considering only congenital heart diseases, this prevalence was $19.2 \%$ (14/73). In $73.7 \%$ of these cases, the cardiac disorder was accessible by the four-chamber view alone. Arrhythmias during obstetrical scan were observed in 26.3 of the babies with prenatal suspicion of a heart abnormality, while only $3.4 \%$ of the patients without prenatal suspicion showed a rhythm alteration $(p=0.009)$. Significant differences between the groups with and without prenatal suspicion of cardiac abnormalities were observed in relation to parity $(p=0.029)$, delivery by cesarean section $(p=0.006)$, need for intensive care $(p=0.046)$ and school education level of the father $(p=0.014)$. At multivariate analysis, only the presence of a rhythm alteration during ultrasound scan was shown to be an independent variable associated with prenatal suspicion of cardiac abnormalities.

Conclusions: routine obstetrical ultrasound has been underused in prenatal screening of congenital heart diseases. Adequate training and making obstetricians and the population a ware of the problem may be instruments for increasing the efficacy of routine obstetrical ultrasound in rising the suspicion of fetal cardiac abnormalities.

KEY WORDS: Ultrasonography. Fetal heart. Congenital defects. Intrauterine diagnosis. 


\section{Referências}

1. Bronshtein M, Zimmer EZ. Prenatal ultrasound examinations: for whom, by whom, what, when and how many? Ultrasound Obstet Gynecol 1997; 10:1-4.

2. Achiron R, Glaser J, Gelernter I, Hegesh J, Yagel S. Extended fetal echocardiographic examination for detecting cardiac malformations in low risk pregnancies. BMJ 1992; 304:671-4.

3. Crane JP, LeFevre ML, Winborn RC, et al. A randomized trial of prenatal ultrasonographic screening: impact on the detection, management, and outcome of anomalous fetuses. Am J Obstet Gynecol 1994; 171:392-9.

4. United States of America. Department of Health and Human Resources. Vital statistics of the United States. $5^{\text {th }}$ ed. Washington: DHHR; 1985.

5. Schirmer M, Colvero MO, Risch JN, Zimmer LP, Zielinsky P. Rastreamento de anormalidades cardíacas fetais durante a ecografia obstétrica. Rev AMRIGS 1997; 41:228-34.

6. Zielinsky P. Malformações cardíacas fetais: diagnóstico e conduta. Arq Bras Cardiol 1997; 69:209-18.

7. Rio Grande do Sul. Secretaria de Saúde. A criança e o adolescente no RS: indicadores de saúde. Porto Alegre: Secretaria da Saúde; 1993. (Indicadores de saúde, 39).

8. Horger EO $3^{\text {rd }}$, Tsai CC. Ultrasound and the prenatal diagnosis of congenital anomalies: a medicolegal perspective. Obstet Gynecol 1989; 74:617-9.

9. Hageman LL. Rastreamento populacional de anormalidades cardíacas fetais por ecocardiografia pré-natal em gestações de baixo risco no município de Porto Alegre [tese]. Porto Alegre: Universidade Federal do Rio Grande do Sul; 2001.

10.Fixler DE, Pastor P, Chamberlin M, Sigman E, Eifler CW. Trends in congenital heart diseases in Dallas County births. 1971-1984. Circulation 1990; 81:137-42.

11.Zielinsky P. Abordagem diagnóstica e terapêutica pré-natal das anormalidades cardíacas fetais. Rev Bras de Ecocardiogr 1992; 17:10-25.
12. Oliveira LT, Bonow FP, Ceccon G, Zimmer LP, Hagemann LL, Zielinsky P. O papel da ultrasonografia obstétrica de rotina no diagnóstico presuntivo de cardiopatias fetais. Rev AMRIGS 1997; 41:181-6.

13.Kirk JS, Riggs TW, Comstock CH, Lee W, Yang SS, Weinhouse E. Prenatal screening for cardiac anomalies: the value of routine addition of the aortic root to the four-chamber view. Obstet Gynecol 1994; 84:427-31.

14.Ott WJ. The accuracy of antenatal fetal echocardiography screening in high and low-risk patients. Am J Obstet Gynecol 1995; 172:1741-9.

15.Stümpflen I, Stümpflen A, Wimmer M, Bernaschek G. Effect of detailed fetal echocardiography as part of routine prenatal ultrasonographic screening on detection of congenital heart disease. Lancet 1996; 348:854-7.

16.Zielinsky P. Alterações do ritmo cardíaco no feto humano: impacto da ecocardiografia pré-natal no diagnóstico e no manejo terapêutico. Rev Bras Ecocardiogr 1991; 4:10-21.

17. Allan LD, Crawford DC, Chita SK, Tynan MJ. Prenatal screening for congenital heart disease. Br Med J (Clin Res Ed) 1986; 292:1717-9.

18.Bromley B, Estroff JA, Sanders SP, et al. Fetal echocardiography: accuracy and limitations in a population at high and low risk for heart defects. Am J Obstet Gynecol 1992; 166:1473-81.

19.Allan LD, Crawford DC, Anderson RH, Tynan MJ. Echocardiographic and anatomical correlations in fetal congenital heart disease. Br Heart J 1984; 52:542-8.

20.Allan LD. Echocardiographic detection of congenital heart disease in the fetus: present and future. Br Heart J 1995; 74:103-6.

21.Benacerraf BR, Pober BR, Sanders SP. Accuracy of fetal echocardiography. Radiology 1987; 165:847-9.

22.Vergani P, Mariani S, Ghidini A, et al. Screening for congenital heart disease with the four-chamber view of the fetal heart. Am J Obstet Gynecol $1992 ; 167: 1000-3$.

23.Zielinsky P. Distúrbios do ritmo cardíaco fetal. Detecção e conduta pré-natal. Arq Bras Cardiol 1996; 66:83-6.

24.Zielinsky P. Papel da cardiologia fetal na atenção primária pré-natal. Arq Bras Cardiol 1994; 63:417-22. 